\title{
EPIDEMIOLOGIA DE UM CASO DE DOENÇA DE CHAGAS NA ILHA DO MOSQUEIRO - PARÁ
}

\author{
Adelson A.A. de Souza, Fernando Tobias Silveira, Michael A. Miles, \\ Marinete M. Póvoa, José Aprígio Nunes Lima e Sebastião Aldo S. Valente.
}

\begin{abstract}
Os autores apresentam os resultados do estudo epidemiológico de um caso autóctone da fase aguda da doença de Chagas na ilha do Mosqueiro, Estado do Pará, aproximadamente $75 \mathrm{~km}$ da capital, Belém. O caso já havia sido objeto de uma publicação anterior. Agora são apresentadas informações epidemiológicas. Nas proximidades da casa do paciente foram capturados em duas palmeiras de Inajá (Maximilian regia) e em uma de Mucajá (Acrocomia sclerocarpia) 114 triatomineos: Rhodnius pictipes, $\mathrm{R}$. robustus, Panstrongylus lignarius, $\mathrm{P}$. geniculatus $e$ Microtriatoma trinidadensis, com tripanossomas em 31 deles. $\mathrm{Na}$ casa do paciente foram encontrados exemplares de Rhodnius pictipes, infectados com formas metaciclicas do Trypanosoma cruzi. Em 14 marsupiais, capturados na localidade, haviam 3 infectados com organismos semelhantes ao T. cruzi A eletroforese dos isoenzimas nos tripanossomas isolados do paciente, de R. pictipes e de Didelphis marsupialis os classificou como zimodema 1. Os autores concluem que a doença de Chagas do paciente teve origem silvestre.
\end{abstract} miologia.

Palavras-chaves: Trypanosoma cruzi. Doença de Chagas. Triatomineo. Epide-

Em 1979 Lainson e cols ${ }^{3}$ quando examinavam 1187 animais silvestres do Estado do Pará, em busca de hematozoários, encontraram parasitos semelhantes ao $T$. cruz $i$ em 13 espécies diferentes, sendo particularmente comuns em uma variedade de marsupiais (Didelphidae), porco-espinho(Coendou prehensilis), tatus (Dasypus novemcinctus) e coati mundi (Nasua nasua). No periodo de 1977 a 1987 , fizemos observações em 1772 animais silvestres de diferentes áreas e foi possivel constatar infecções por $T$. cruzi em uma grande variedade de animais, além dos acima citados 15 . Até o momento existem descritos 10 casos autóctones de doença de Chagas no Estado do Pará, a partir dos primeiros descritos por Shaw e col ${ }^{12}$.

O Inquérito Sorológico Nacional sobre a doença de Chagas, realizado pela Superintendência de Campanhas de Saúde Pública do Ministério da Saúde (SUCAM), no periodo de 1975-1980, revelou que a prevalência da doença de Chagas, no Estado do Pará, era de $0,5 \%$ em 29.824 amostras processadas no laboratório do Instituto Evandro Chagas 2 . Segundo Lainson e $\mathrm{col}^{3}$, não existe evidência de espécies de barbeiros silvestres (Hemiptera: Reduviidae) colonizando casas, porém, ocasionalmente, adultos de $P$.

\footnotetext{
Departamento de Parasitologia do Instituto Evandro Chagas - Fundaçao Serviço Especial de Saúde Pública (SESP) e Departamento de Protozoologia da London School of Hygiene and Tropical Medicine.

Suporte financeiro fornecido pela Wellcome Trust e Fundação SESP.
}

Recebido para publicação em 28/1/88. geniculatus e $R$. pictipes foram encontrados em casas no subúrbio próximo a florestas. A doença de Chagas poderá tornar-se endêmica na bacia Amazônica, se os triatomineos silvestres forem capazes de adaptar-se às habitações domésticas; porém o perigo maior está na possibilidade da importaçao de vetores já domiciliados ao longo das grandes rodovias procedentes de outras regiōes endêmicas. Vários métodos foram empregados para identificar espécies de triatomineos silvestres e seus ecótopos naturais nos arredores de Belém, Estado do Pará7. Dez espécies foram encontradas: Rhodnius pictipes, $R$. robustus, $R$. paraensis, Pastrongylus lignarius, $P$. geniculatus, $P$. rufotuberculatus, Eratyrus mucronatus, Microtriatoma trinidadensis, Belminus herreri e Triatoma rubrofasciata.

Apesar de todas essas evidências, da transmissão silvestre do $T$. cruzi, não se conhece, até o momento, vetores domiciliares do $T$. cruz $i$ em toda a bacia Amazônica ${ }^{7}$. É sabido que alguns exemplares adultos desses triatomineos podem, ocasionalmente, voar a distância razoável ${ }^{3}$, podendo localizar-se no interior das casas. Tal fato pode ocorrer com Rhodnius pictipes, Panstrongylus geniculatus, Triatoma maculata e Eratyrus mucronatus no Estado do Amazonas e com $R$. pictipes, $P$. geniculatus e $P$. lignarius no Estado do Pará.

\section{MATERIAL E MÉTODOS}

Localidade de estudos - Este trabalho discute um caso autóctone de doença de Chagas, de origem silvestre, na Ilha do Mosqueiro, Estado do Pará, 
distante aproximadamente $75 \mathrm{~km}$ da capital, Belém. Nossos estudos epidemiológicos complementam o diagnóstico do "Nono caso autóctone de doença de Chagas registrado no Pará" por Silveira e col ${ }^{14}$ em 1979.

\section{Investigação e dissecação de palmeiras}

Esse método é desenvolvido através'da dissecação de possiveis refúgios de animais, tais como a copa de palmeiras. As palmeiras são derrubadas e dissecadas inteiramente, atraves do auxilio de motosserra, luvas de couro, terçados, pinças e roupas especiais, requerendo bastante atenção por parte dos pesquisadores para a captura de triatomíneos e de algum animal que nela faça morada. $O$ critério para selecionar palmeiras que serão derrubadas e dissecadas requer, pelas nossas observações, que elas sejam de preferência sujas, possibilitando assim melhores condições de abrigo para os animais. No trabalho de dissecação de palmeiras, de um modo geral utilizou-se motosserra STHIL modelos 51 e 8 . Os triatomíneos capturados são acondicionados em copos plásticos com tampa perfurada, possibilitando a entrada de ar, sendo usado, geralmente, um copo para cada palmeira.

\section{Captura e seguimento de mamíferos}

Para captura de mamiferos foram utilizadas armadilhas dobráveis, feitas de arames (Kinro Products Ltd.), tendo como isca a mistura de pasta de amendoim, ração, óleo de dendê, banana e um pequeno pedaço de jaca ou abacaxi, bem como um pequeno pedaço de milho, principalmente para roedores. Pelas nossas observações, essa isca tem grande aceitação pelos animais, além de possuir um odor muito ativo e atraente.

Para segui-los, foi utilizado um método simples de seguir mamiferos, criação de Miles ${ }^{4}$ e Miles e cols 7 . Consta de um tubo de plástico contendo em seu interior linhas de variadas dimensōes, e, por esta razão foram denominadas tipo A (2300 metros), B (1300 metros), C (280 metros), D (160 metros) e E (1350 metros), constituindo diferentes aparelhos, com diferentes pesos e para determinados portes de animais. Os aparelhos são colocados no dorso ou no lado do animal anestesiado com Sagatal (May-Baker Ltd. Dagenham) ou Ketalar (Parke Davis), geralmente no fim da tarde, sendo soltos ao anoitecer, tendo a extremidade da linha amarrada em árvore próxima da armadilha, onde foram capturados. No dia seguinte, a linha é seguida, objetivando identificar-se os ecótopos e examinar seus ninhos para captura de barbeiros. Em geral, os ninhos são trazidos em sacolas plásticas para melhores investigaçōes no laboratório, enquanto os aparelhos são pesados novamente para determinar a distância percorrida na floresta.

\section{Armadilha de luz}

Colocamos duas armadilhas entre duas palmeiras de Mucajá (Acrocomia sclerocarpa), de aproximadamente 15 metros de altura e distante 30 metros da residência do paciente que apresentou a fase aguda da doença de Chagas, tendo como fonte de energia um gerador Honda modelo E 300 que era ligado ao anoitecer e desligado ao amanhecer. Essas observaçōes foram realizadas durante cinco noites.

\section{Xenodiagnóstico}

Esse método parasitológico foi utilizado como tentativa de confirmação de diagnóstico e isolamento de cepa para posterior caracterização bioquímica por eletroforese de enzimas.

\section{Hemocultura}

Método que tem sido utilizado ao lado do xenodiagnóstico para o diagnóstico parasitológico da tripanosomíase americana. Utilizamos o meio bifásico 5 .

\section{Eletroforese de enzimas}

A eletroforese de isoenzimas foi utilizada como método de caracterizaçâo das cepas de $T$. cruzi, de acordo com Miles e cols 910 .

\section{RESULTADOS}

Informaçōes sobre o caso: SBS, 19 anos, sexo masculino, pardo, solteiro, estudante, natural do Pará, procedente de Vila do Mosqueiro, onde reside desde o nascimento, foi internado na Enfermaria São Miguel no dia 11.09.79, acusando, como sintoma principal, febre elevada, há 11 dias, de caráter contínuo, acompanhada desde o início de calafrios intermitentes e mal-estar geral. $O$ exame físico revelou, entre outras coisas, figado palpável a $1 \mathrm{~cm}$ do rebordo costal direito, superficie lisa, bordos arredondados e moderadamente dolorosos à palpação profunda; baço palpável a $1 \mathrm{~cm}$ do rebordo costal esquerdo, consistência normal e pouco doloroso à palpação profunda; micropoliadenopatia, não dolorosa, de localização cervical, retroauricular, axilar e inguinal. $\mathrm{O}$ exame direto do sangue, a fresco e em esfregaço corado pela Giemsa, apresentou parasito com morfologia semelhante ao $T$. cruzi. O xenodiagnóstico com leitura após 30 dias apresentou resultado positivo. A imunofluorescência 
indireta para doença de Chagas foi positiva: 1:160 (IgM) e 1:160 (IgG).

Foram capturados, no interior da residência do paciente, três exemplares de Rhodnius pictipes, sendo dois machos e uma fêmea, os quais foram dissecados, $\mathrm{e}$ ao microscópio foram observados organismos diferentes ao T. cruzi.

Investigação e dissecação de palmeiras: Nessa fase do trabalho foram dissecadas duas palmeiras de Inajá (Maximilian regia) e uma de Mucajá (Acrocomia sclerocarpa) localizadas às proximidades da residência, e um total de 114 triatomineos foram capturados entre adultos e ninfas de 5 espécies diferentes, Rhodnius pictipes, Rhodrius robustus, Panstrongylus lignarius, $P$. geniculatus e Microtriatoma trinidadensis (Tabela 1).

Captura e seguimento de mamiferos: $\mathrm{Na}$ área próxima à residência do paciente, foram capturados um Caluromys philander e um Didelphis marsupialis. Em outra área de estudo epidemiológico foram capturados 19 animais (Tabela 2). Foram soltos e seguidos 14 animais com diferentes tipos de linha, sendo recuperados um total de $7 \mathrm{com}$ uma taxa de recuperação de $50 \%$. Os ninhos examinados não continham nenhum triatomíneo.

Armadilha de luz: Foram capturados 3 triatomineos, 1 exemplar de $P$. geniculatus (macho) e 2 exemplares de $R$. pictipes (macho). Observamos que nas duas últimas noites essas armadilhas foram prejudicadas pelo mal tempo, com a ocorrência de muita chuva e fortes ventos.

Xenodiagnóstico: Foram realizados 10 xenodiagnósticos entre os familiares do paciente, tendo todos resultados negativos.

Estudo de enzimas: Nas presentes observaçōes desse nono caso autóctone do Estado do Pará, o trabalho de eletroforese de enzimas demonstrou que os tipos enzimáticos de Trypanosoma cruz $i$ identificados foram semelhantes, tanto no tripanossoma isolado no paciente como nos $R$. pictipes e $D$. marsupialis, examinados, sendo tais amostras caracterizadas como zimodema tipo I (Figura I).

Tabela 1 - Infecção por tripanossomas semelhantes ao T. cruzi na Ilha do Mosqueiro

\begin{tabular}{llcc}
\hline Triatomineos & Estágio & Quantidade & Infectados \\
\hline R. pictipes & Adultos & 29 & 20 \\
& Ninfas -V & 15 & - \\
& IV & 4 & - \\
& III & 17 & - \\
II & 11 & - \\
R. robustus & I & 9 & 10 \\
$M$. trinidadensis & Adultos & 23 & - \\
Adultos & Ninfas - II & 3 & - \\
$P$. lignarius & Adultos & 1 & - \\
\hline Total & Ninfas - III & 1 & - Armadilha de luz - \\
& & 1 & 31 \\
\hline
\end{tabular}

Obs: 2 exemplares de $R$. pictipes foram capturados em armadilha de luz.

Tabela 2- Infecção por tripanossomas semelhantes ao T. cruzi em animais capturados na Ilha do Mosqueiro.

\begin{tabular}{lcc}
\hline Animais capturados & Quantidade & Infectados \\
\hline$P$. opossum & 6 & 1 \\
D. marsupialis & 8 & 2 \\
M. nudicaudatus & 4 & 0 \\
Oryzomys sp. & 1 & 0 \\
$P$. guyannensis & 2 & 0 \\
\hline Total & 21 & 3
\end{tabular}


Souza AAA, Silveira FT, Miles MA, Póvoa MM, Lima JAN, Valente SAS. Epidemiologia de um caso de Doença de Chagas na Ilha do Mosqueiro-Pará. Revista da Sociedade Brasileira de Medicina Tropical 21:187-192, Out-Dez, 1988
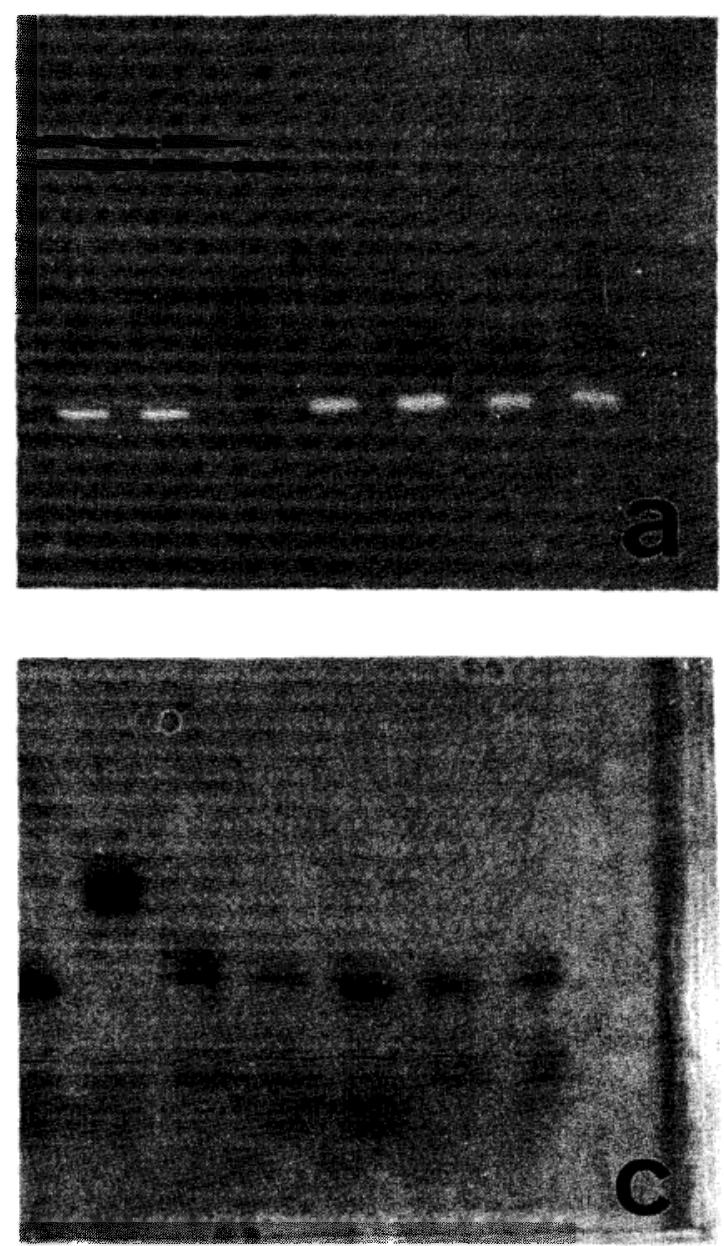

Figura I - Placas de eletroforese contendo a amostra de $S B S$, configurando o zimodema 1 , o mesmo das amostras de R. pictipes $e$ D. marsupialis. As enzimas mostradas são: a) $A S A T$; b) $A L A T$; c) GPI e d) PGM.

Em cada placa as amostras de trypanosoma são (da

\section{DISCUSSÃO}

Após vários anos de trabalho de campo, em diversas áreas estudadas, chamamos a atenção para a importancia que as palmeiras, das espécies Acrocomia sclerocarpa (mucajá), Maximilian regia (inajá), Orbignia speciosa (babaçu), Jessenia bataua (patauá) e Mauritia flexuosa (miriti), oferecem como fonte de casos esporádicos da doença de Chagas na região Amazônica, por apresentarem, segundo nossas investigações, um percentual elevado de infestação por triatomineos.

Acreditamos que tenhamos detectado os pontos cardeais associados à cadeia epidemiológica, da doença de Chagas, identificando os vetores associados, bem como os prováveis animais silvestres implicados nesse caso agudo autóctone do Estado do Pará.
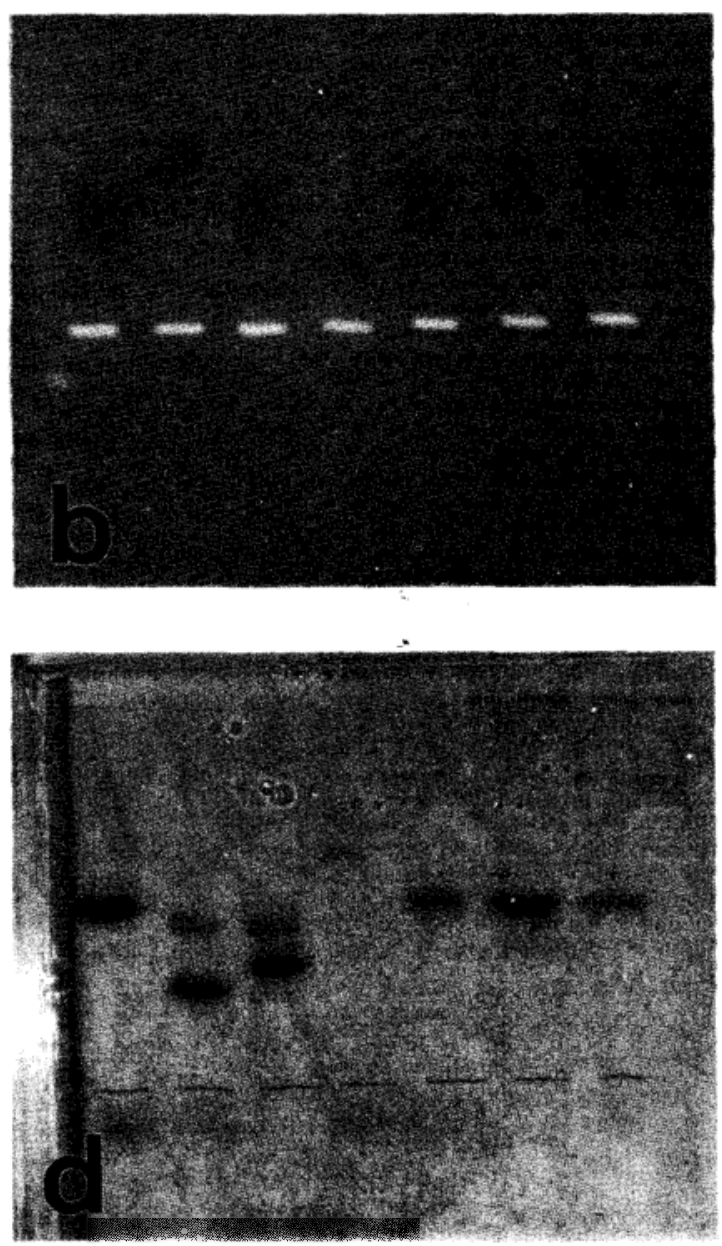

esquerda para direita): I-WA 250 clone 2, padrâo de $Z 1$; 2-Esmeraldo clone 3, padrão de Z2; 3-CAN III clone 1, padrão de Z3; 4-R 1625, Trypanosoma rangeli do homem, El Salvador; 5 - X 10 clone 1, amostra do paciente $S B S$; 6-Bug 4207, R. pictipes e 7-Caixa 1078, D. marsupialis.

A captura e identificação de animais silvestres possibilitaram o isolamento de cepas de $T$. cruz $i$, que foram caracterizados por eletroforese de enzimas como zimodema tipo I, o mesmo perfil enzimático que ofereceram os isolamentos de cepas de triatomíneos e a cepa do paciente estudado, mostrando claramente que a origem desse caso foi originariamente silvestre, pela completa ausência do zimodema II, prevalente nas áreas endêmicas de transmissão doméstica. As populações de parasitos identificados por perfis enzimáticos idênticos têm sido chamadas de zimódema por Miles e cols ${ }^{7}$. Os estudos de enzimas realizados na bacia Amazônica demonstram que os zimodemas I e III são prevalentes entre os mamiferos e triatomineos do Estado do Pará, tendo sido responsáveis pelos primeiros casos autóctones de doença de Chagas na cidade de Belém, confirmando a origem local desses $\operatorname{casos}^{6}$. 
Souza AAA, Silveira FT, Miles MA, Póvoa MM, Lima JAN, Valente SAS. Epidemiologia de um caso de Doença de Chagas na Ilha do Mosqueiro-Pará. Revista da Sociedade Brasileira de Medicina Tropical 21: 187-192, Out-Dez, 1988

Ressaltamos também que, desde 1970 , o método de eletroforese de enzimas tem se revelado importante com largo emprego na taxonomia zoológica por constituirem-se, as enzimas, excelentes marcadores genéticos que vieram possibilitar o entendimento da epidemiologia e biologia básicas de tripanossomas e leishmânias. Por essa razão, o uso das enzimas, como marcadores genéticos, tem crescido de maneira acentuada nos últimos anos. Ready e Miles ${ }^{11}$, demonstraram a aplicação da eletroforese de enzimas na taxonomia numérica, quando detectaram três zimodemas de $T$. cruzi do Brasil, os quais foram claramente definidos e separados por caracteres enzimáticos. Recursos para o diagnóstico da doença de Chagas ficaram bastante evidenciados através das técnicas de hemocultura do sangue dos animais 4 bem como através da cultura das fezes dos triatomíneos dissecados e que apresentaram positividade com formas metaciclicas do T. cruzi. Em se tratando de diagnóstico da doença de Chagas, estudo comparativo entre a hemocultura e o xenodiagnóstico foi realizado por vários pesquisadores e a maioria dos resultados permitiu concluir que o método mais sensivel foi o xenodiagnóstico. Para captura de vetores triatomineos, o uso de armadilha de luz tem se revelado altamente produtivo. No Panamá, Whitlaw e Chaniotis 16 , demonstraram sua eficiência, pois capturaram 7 de 8 espécies, conhecidas naquele pais. Foi demonstrada também uma diferença de captura em diferentes estações com 0,56 triatomineos, por noite, nas estações secas, e 0,26 para as estações chuvosas. Em nossos trabalhos, na bacia Amazônica, tivemos oportunidade de capturar exemplares de 7 espécies: $R$. pictipes, $P$. geniculatus, $E$. mucronatus, $P$. lignarius, $P$. nufotuberculatus, $R$. robustus e Microtriatoma trinidadensis. A partir das nossas observaçōes, recomendamos um trabalho de pesquisa mais profundo, associado à vigilância epidemiológica e uso de inseticidas nas espécies de palmeiras citadas, principalmente aquelas localizadas na cidade de Belém e em sua periferia, objetivando evitar novos casos semelhantes a este aqui descrito.

\section{SUMMARY}

The authors present the results of an epidemiological study relating to a case of acute Chagas' disease acquired in the island of Mosqueiro, State of Para, approximately $75 \mathrm{~km}$ from the capital Belem. The patient has been the object of a previous publication but now epidemiological information is reported. Near the house of the patient in two Inaja palm trees (Maximilian regia) and one Mucaja palm (Acrocomia sclerocarpia) 114 triatomine bugs were captured of the following species: Rhodnius pictipes, R. robustus, Panstrongylus lignarius, $\mathrm{P}$. geniculatus and $\mathrm{Mi}-$ crotriatoma trinidadensis. Trypanosomes were found in 31 bugs. In the house of the patient specimens of $\mathrm{R}$. pictipes were captured infected with metacyclic forms of Trypanosoma cruzi. In 14 marsupials captured in the locality three had infections with cruzi like trypanosomes. Enzyme electrophoresis of the trypanosomes isolated from the patient, $\mathrm{R}$. pictipes and Didelphis marsupialis were classified as zymodeme 1. The authors conclude that the patients Chagas' disease was of sylvatic origin.

Key-words: Trypanosoma cruzi. Chagas' disease. Triatomids. Epidemiology.

\section{AGRADECIMENTOS}

Agradecemos a decisiva colaboração de nossos auxiliares Francisco dos Santos Gomes e Raimundo Nivaldo de Almeida, bem como do Sr. Paulo Barbosa da Silva pelos contatos, facilidades e informaçōes prestadas na Ilha do Mosqueiro. Ao dr. Habib Fraiha Neto, entomologista da IEC pela identificação dos triatomíneos capturados. A Sra. Maria das Graças Soares da Silva pelo serviço datilográfico e ao Sr. Aluizio de Figueiredo Silva pelo serviço fotográfico.

\section{REFERÊNCIAS BIBLIOGRÁFICAS}

1. Brener Z, Andrade Z. Trypanosoma cruzi e doença de Chagas. Rio de Janeiro, Guanabara Koogan, 1979.

2. Camargo ME, Silva GR, Castilho EA, Silveira AC. Inquérito sorológico da prevalência de infecção chagásica no Brasil, 1975-1980. Revista do Instituto de Medicina Tropical de São Paulo 26: 192-204, 1984.

3. Lainson R, Shaw JJ, Fraiha H, Miles MA, Draper CC. Chaga's disease in the Amazon Basin: I. Trypanosama cruz $i$ infections in silvatic mammals, triatomine bugs and man in the State of Para, north Brazil. Transactions of the Royal Society of Tropical Medicine and Hygiene 73: 193-204, 1979.

4. Miles MA. A simple method of tracking mammals and locating triatomine vectors of Trypanosoma cruzi in Amazonian forest. The American Journal of Tropical Medicine and Hygiene 25: 671-674, 1976.

5. Miles MA, Lanham SM, Souza AA, Póvoa M. Further enzymic characters of Trypanosoma cruzi and their evaluation for strain identification. Transactions of the Royal Society of Tropical Medicine and Hygiene 74: 221-237, 1980.

6. Miles MA, Povoa MM, Souza AA, Lainson R, Shaw JJ, Ketteridge DS. Chagas's disease in the Amazon Basin: II. The distribution of Trypanosoma cruzi zymodemes 1 and 3 in Para State, north Brazil. Transactions of the Royal Society of Tropical Medicine and Hygiene 75 : 667-674, 1981.

7. Miles MA, Souza AA, Póvoa M. Chagas's disease in the Amazon Basin: III. Ecotopes of ten triatomine bug species (Hemiptera: Reduviidae) from the vicinity of Belem, Para, State, Brazil. Journal of Medical Entomology 18: 266-278, 1981. 
8. Miles MA, Souza AA, Póvoa MM. Mammal tracking and nest location in Brazilian forest with an improved spool-and-line device. Journal of Zoology 195: 331-347, 1981.

9. Miles MA, Souza AA, Póvoa M, Shaw JJ, Lainson R, Fraiha H. Três tipos distintos de Trypanosoma cruzi identificados por eletroforese de enzimas, infetam o homem m no Brazil. Proceedins of the XIV Congress of the Brazilian Society of Tropical Medicina, João Pessoa, Brazil, 1978.

10. Miles MA, Souza A, Póvoa M, Shaw JJ, Lainson R, Toye PJ. Isozymic heterogeneity of Trypanosoma cruzi in the first autochthonous patients with Chagas' disease in Amazonian. Nature 272: 819-821, 1978.

11. Ready PD, Miles MA. Delimitation of Trypanosoma cruzi zymodemes by numerical taxonomy. Transactions of the Royal Society of Tropical Medicine and Hygiene 74: 238-242, 1980.

12. Shaw JJ, Lainson R, Fraiha $H$. Consideraçōes sobre a epidemiologia dos primeiros casos autóctones de doença de Chagas registrados em Belém, Pará, Brasil. Revista de Saúde Pública 3: 153-157, 1969.

13. Sherlock IA, Guitton N, Miles M. Rhodnius paraensis, espécie nova do Estado do Pará- Brasil - (Hemiptera: Reduviidae, Triatomine). Acta Amazônica 7: 71-74, 1977.

14. Silveira FT, Dias MGV, Pardal PP, Lobão AO, Melo GB. Nono caso autóctone de Doença de Chagas registrado no Estado do Pará, Brasil (nota prévia). Hiléia Médica 1: 61-62, 1979.

15. Souza AA, Miles MA, Póvoa M, Rodrigues IC, Carvalho RA, Valente SAS. Tripanosomiases humanas, Trypanosoma cruzi e doença de Chagas. Trypanosoma rangeli. Instituto Evandro Chagas; 50 anos de contribuição às cièncias biológicas e à Medicina Tropical, Belém, Fundaçào Serviços de Saúde Pública 1986.

16. Whitlaw JT, Chaniotis BN. Palm trees and Chagas' disease in Panama. The American Joumal of Tropical Medicine and Hygiene 27: 873-881, 1978. 